\title{
Circadian rhythms: data analysis and mathematical modeling
}

\author{
N.L. Podkolodnyy ${ }^{1,2 *}$, N.N. Tverdohkleb ${ }^{1,3}$, O.A. Podkolodnaya ${ }^{1}$ \\ ${ }^{1}$ Institute of Cytology and Genetics SB RAS, Novosibirsk, Russia \\ ${ }^{2}$ Institute of Computational Mathematics and Mathematical Geophysics SB RAS, Novosibirsk, Russia \\ ${ }^{3}$ Novosibirsk State University, Novosibirsk, Russia \\ *e-mail:pnl@bionet.nsc.ru
}

Key words: circadian rhythm, mathematical modelling, gene expression analysis, GO enrichment analysis, biological processes

Motivation and Aim: A large-scale analysis of the circadian dynamics of transcriptomes and translatomes together with gene networks and protein-protein interaction network makes it possible to identify the molecular processes with circadian rhythm and to reconstruct the structure of the mathematical model for circadian regulation of biologically important molecular processes. The purpose of this work is to develop such kind methods and to apply them for modeling the mutual influence of the circadian oscillator and the NAD+ / SIRT1 system.

Methods and Algorithms: We used experimental data on the gene expression at the mRNA level and ribosome profiling (GSE67305 and GSE81283) in mouse liver and kidney. Identification of circadian patterns of gene expression was carried out by the methods of correlation, cluster analysis and the principal component analysis. The modeling and simulation were performed in Matlab (ODE solver ode15s).

Results: We have identified genes that demonstrate pronounced circadian dynamics of transcription and translation. Based on the cluster analysis of the expression data, we identified the groups of genes with different circadian dynamic pattern of transcription and translation and the groups of genes with different circadian phase characteristics.

For these groups of genes the GO enrichment analysis was carried out. As a result, we have identified molecular processes with circadian regulation and the groups of genes with significantly different temporal phase patterns in mouse liver and kidney. We have reconstructed and analyzed the gene network of mammalian circadian clocks. We identified the central component of circadian rhythm regulation and the functional subsystems interacting with it. This makes it possible to simulate circadian regulation of functionally important molecular processes in a mammalian cell. Using these approaches, a simulation of the mutual influence of the circadian oscillator and the NAD+ / SIRT1 system (cell energy balance regulator and some kind of "metabolic oscillator") was carried out. Based on the experimental data on the changes in the activity of SIRT1 and the level of NAD+ with age, we investigated the effect of these age-related changes on the functioning of the circadian oscillator. Mathematical modeling has shown that the age-related decline in SIRT1 activity may be one of the causes of disturbances in the functioning of the circadian oscillator in suprachiasmatic nuclei, which may also lead to disturbances in the circadian rhythms of the organism as a whole.

Acknowledgements: Supported by Presidium of SD RAS (No. 0324-2018-0021) and by RF Government (No. 0324-2018-0017). 